\title{
FEM Modeling of Nanocomposites Low Speed Impact Behavior
}

\author{
Saud Aldajah, Yousef Haik, Kamal Moustafa, and Ammar Alomar
}

\begin{abstract}
A 2D finite element model using ABAQUS has been developed in order to understand the role of nanoclay in enhancing the low speed impact behavior of laminated woven kevlar nanocomposite. The model simulates an impactor as a shell rigid body while the composite samples were simulated in two dimensional model. ABAQUS/Explicit 6.8 was used to simulate the composite samples in this study. The FEM analysis focuses on the stress distribution in the impacted composite samples; the ability of the impactor to penetrate the samples; and the expected failure mode based on the Hashin's theory damage initiation criteria for fiber- reinforced composites. The damage evolution was determined in terms of displacement and/or energy. In the displacement damage evolution, it is possible to define the damage as a function of the total or the plastic displacement after damage initiation. While as, in the energy evolution damage, it is defined in terms of the energy required for failure (fracture energy) after the initiation of damage. The theoretical and experimental results were in good agreement..
\end{abstract}

Index Terms-FEM modeling, nanocomposite, low speed impact.

\section{INTRODUCTION}

In the finite element method, a structure is broken down into many small simple blocks or elements. The behavior of an individual element can be described with a relatively simple set of equations. Just as the set of elements would be joined together to build the whole structure, the equations describing the behaviors of the individual elements are joined into an extremely large set of equations that describe the behavior of the whole structure. The computer can solve this large set of simultaneous equations. From the solution, the computer extracts the behavior of the individual elements. From this, it can get the stress and deflection of all the parts of the structure. The stresses are compared to allowed values of stress for the materials to be used, to see if the structure is strong enough [1].

Realistic finite element method (FEM) problems might consist of up to hundreds of thousands, and even several millions, of elements and nodes, and therefore they are usually solved in practice using commercially available software packages. There are currently a large number of commercial software packages available for solving a wide range of problems: solid and structural mechanics, heat and

Manuscript received June 4, 2013; revised November 13, 2013.

Saud Aldajah, Yousef Haik, and Kamal Moustafa are with the Mechanical Engineering Department, United Arab Emirates University, P. O. Box 15551 Al-Ain, UAE (e-mail: s.aldajah@uaeu.ac.ae)

Ammar Alomar is with the Tawazun Precision Industries, Tawazun Industrial Park, P. O. Box 129862, Abu Dhabi, U.A.E. mass transfer, fluid mechanics, acoustics and multi-physics, which might be static, dynamic, linear and nonlinear. Most of these software packages use the finite element method, or are used in combination with other numerical methods. All these software packages are developed based on similar methodology, with many detailed and fine-tuned techniques and schemes [2]. ABAQUS is a powerful finite element software package. It is used in many different engineering fields throughout the world. ABAQUS performs static and/or dynamic analysis and simulation on structures. It can deal with bodies with various loads, temperatures, contacts, impacts, and other environmental conditions.

ABAQUS software has many different modules, our analysis carried out using ABAQUS/ Explicit finite element software (version 6.8). There are other modules in the ABAQUS finite element package, including ABAQUS/standard, ABAQUS/Implicit ABAQUS/CAE and ABAQUS/Viewer. ABAQUS/Explicit is mainly used for explicit dynamic analysis. ABAQUS/CAE is an interactive preprocessor that can be used to create finite element models and the associated input file for ABAQUS. ABAQUS/Viewer is a menu-driven interactive post-processor for viewing the results obtained from ABAQUS/Standard and ABAQUS/Explicit. In this research, however, the focus will be on the writing of the ABAQUS/Explicit input file, and ABAQUS/Explicit will from now on just be called ABAQUS.

\section{MATERIALS MODELS}

\section{A. Kevlar Plates}

The material properties in ABAQUS defined as an embedded property for a proper "section", then the mechanical part will be assigned to this section. After this step, we can consider every geometrical part had been attached to it is material properties. Composite parts need to be assigned with proper section, ABAQUS library has specialized section to define "composite shells". This section is capable of defining the material property, the orientation, the thickness and the number of integration points of each layer. Shell section behavior is defined in terms of the response of the shell section to stretching, bending, shear, and torsion. Composite shell sections are composed of layers made of different materials in different orientations.

30-Layers were defined to capture the response of 15 woven Kevlar layers, the 30 layers arranged in [0/90/45/135] symmetrically to replace 15 woven layers arranged in [0/45] orientation, vinylester layers were defined between Kevlar layers [3].

Shell sections integrated during analysis allow the 
cross-sectional behavior to be calculated by numerical integration through the shell thickness, thus providing complete generality in material modeling. Any number of material points can be defined through the thickness, and the material response can vary from point to point. This type of shell section is generally used with nonlinear material behavior in the section.

4-node doubly curved general-purpose shell, reduced integration S4R element was used to model the two dimensional composite elements, this element capable of capturing the nonlinear response of the Kevlar material, Fig. 1.

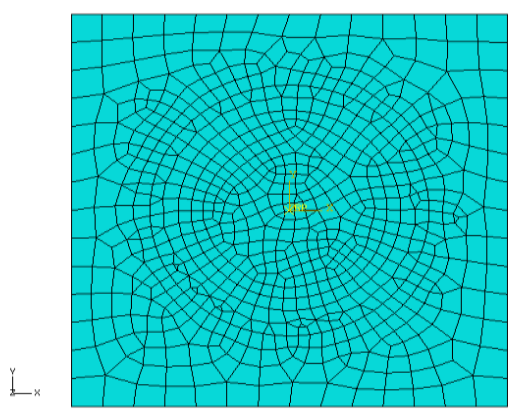

Fig. 1. Meshed kevlar plate

\section{B. Impactor}

The IMPACTOR didn't show any deformation during the experimental tests, because of this it is logical to model the impactor with rigid elements. Rigid elements can be used to define the surfaces of rigid bodies for contact. ABAQUS doesn't need to define material type or element type for rigid bodies, and we still can simulate a rigid projectile penetrating meshed area with nodes.

\section{MATERIALS PROPERTIES}

Kevlar has orthotropic elastic properties [4], Linear elasticity in an orthotropic material is most easily defined in ABAQUS by giving the "engineering constants": the three moduli $E_{1}, E_{2}, E_{3}$; Poisson's ratios $v_{12}, v_{13}, v_{23}$; and the shear moduli $G_{12}, G_{13}$, and $G_{23}$ associated with the material's principal directions. These moduli define the elastic compliance according to

$$
\left\{\begin{array}{l}
\varepsilon_{11} \\
\varepsilon_{22} \\
\varepsilon_{33} \\
\gamma_{12} \\
\gamma_{13} \\
\gamma_{23}
\end{array}\right\}=\left[\begin{array}{cccccc}
1 / \mathrm{E}_{1} & -v_{21} / \mathrm{E}_{2} & -v_{31} / \mathrm{E}_{3} & 0 & 0 & 0 \\
-v_{12} / \mathrm{E}_{1} & 1 / \mathrm{E}_{2} & -v_{32} / \mathrm{E}_{3} & 0 & 0 & 0 \\
-v_{13} / \mathrm{E}_{1} & -v_{23} / \mathrm{E}_{2} & 1 / \mathrm{E}_{3} & 0 & 0 & 0 \\
0 & 0 & 0 & 1 / G_{12} & 0 & 0 \\
0 & 0 & 0 & 0 & 1 / G_{13} & 0 \\
0 & 0 & 0 & 0 & 0 & 1 / G_{23}
\end{array}\right] \quad\left\{\begin{array}{l}
\sigma_{11} \\
\sigma_{22} \\
\sigma_{33} \\
\sigma_{12} \\
\sigma_{13} \\
\sigma_{23}
\end{array}\right\}
$$

The quantity $v_{i j}$ has the physical interpretation of the Poisson's ratio that characterizes the transverse strain in the $j$-direction, when the material is stressed in $i$-direction. In general, $v_{i j}$ is not equal to $v_{j_{i}}$ : they are related by

$$
v_{i j} / E_{i}=v_{j_{i}} / E_{j}
$$

The engineering constants can also be given as functions of temperature and other predefined fields, if necessary (ABAQUS).

The following mechanical properties were used for Kevlar 49: $E_{1}=129.6 \mathrm{GPa}, E_{2}=2.49 \mathrm{GPa}, \quad E_{3}=2.49 \mathrm{GPa}, v_{12}=0.35$ $\mathrm{GPa}, v_{13}=0.35 \mathrm{GPa}, v_{23}=0.31 \mathrm{GPa}, G_{12}=2.01 \mathrm{GPa}, G_{13}=$ $2.01 \mathrm{GPa}, G_{23}=2.01 \mathrm{GPa}$ [4].

While for Vinylester they were $E=2.34 \mathrm{GPa} \& v=0.33$ $[5]$.
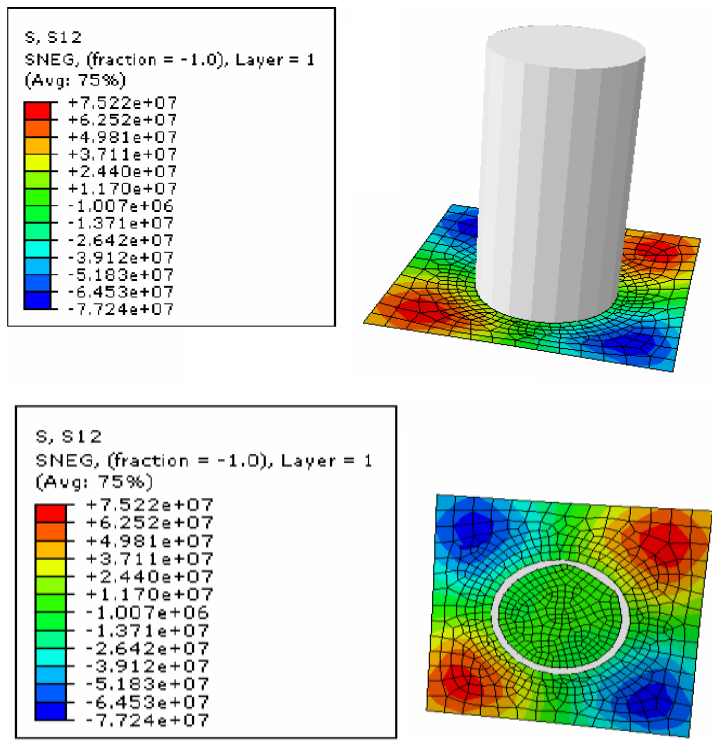

Fig. 2. Shear stress $\sigma_{12}$ in the $\mathrm{X}-\mathrm{Y}$ direction of the control sample.
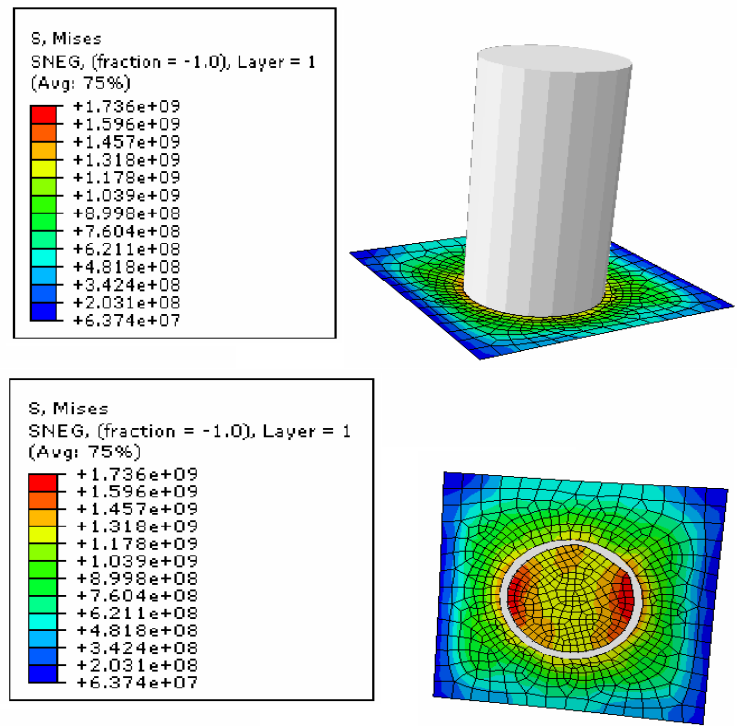

Fig. 3. Von mises stress failure criterion of the control sample
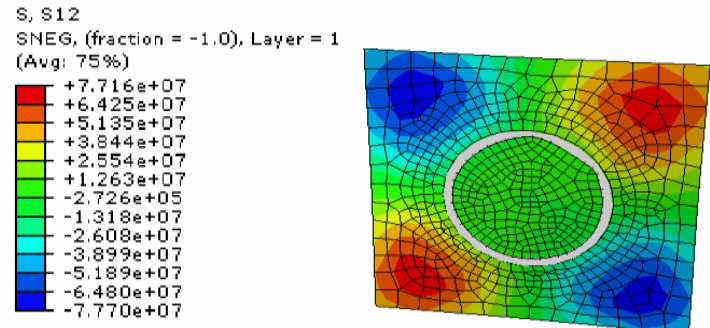

Fig. 4. Shear stress $\sigma_{12}$ in the X-Y direction of nanoclay reinforced sample

\section{UNITS}

Use either SI (MKS) or CGS as primary units. (SI units are 
strongly encouraged.) English units may be used as secondary units (in parentheses). This applies to papers in data storage. For example, write " $15 \mathrm{~Gb} / \mathrm{cm}^{2}\left(100 \mathrm{~Gb} / \mathrm{in}^{2}\right)$." An exception is when English units are used as identifiers in trade, such as " $3 \frac{1}{2}$ in disk drive." Avoid combining SI and CGS units, such as current in amperes and magnetic field in oersteds. This often leads to confusion because equations do not balance dimensionally. If you must use mixed units, clearly state the units for each quantity in an equation.

The SI unit for magnetic field strength $H$ is $\mathrm{A} / \mathrm{m}$. However, if you wish to use units of $\mathrm{T}$, either refer to magnetic flux density $B$ or magnetic field strength symbolized as $\mu_{0} H$. Use the center dot to separate compound units, e.g., "A. $\mathrm{m}^{2}$."

\section{RESULTS AND DISCUSSION}

\section{A. Control Sample}

More than four hours were needed to the solution to converge, the results were able to till about the deformed shape, the different stress tensor components, the different strain tensor components, the different stress and strain failure criterion, displacements in all directions, velocity, acceleration, reaction forces, compression pressure and many other parameters. The shear stress contours in the control sample at the final step was shown in Fig. 2. The values of stresses exceeded the interlaminar shear stress, and this means that the delamination between the Kevlar layers will take place during the penetration process.

Fig. 3 shows the Von Mises Stress distribution in the control sample at the final step. The stress level exceeded the fiber fracture strength at the circumference of the contact circle. This will allow the impactor to penetrate the Kevlar layers

In Abaqus the damage initiation criteria for fiberreinforced composites are based on Hashin's theory (Hashin and Rotem [6] and Hashin [7]). These criteria consider four different damage initiation mechanisms: fiber tension, fiber compression, matrix tension, and matrix compression.

\section{B. Sample with Nano Particles (2 \% of Nanoclay)}

The only difference between the control sample model and this model is the mechanical properties of the composite matrix, the effect of the nanoclay on the bulk of the composite was modeled by modifying the modulus of elasticity of the matrix to be $2.58 \mathrm{GPa}$ and the poisson's ratio equal 0.33 .

The shear stress contours at the final step in the nanoclay reinforced sample was shown in Fig. 4. The values of stresses exceeded the interlaminar shear stress, and this means that the delamination between the Kevlar layers will take place during the penetration process, the maximum shear stress was very close to the maximum shear stress in the control sample at the same time increment

Fig. 5 shows the Von Mises Stress distribution in the nanoclay reinforced kevlar sample at the final step. The stress level exceeded the fiber fracture strength at the circumference of the contact circle. This will allow the impactor to penetrate the Kevlar layers.
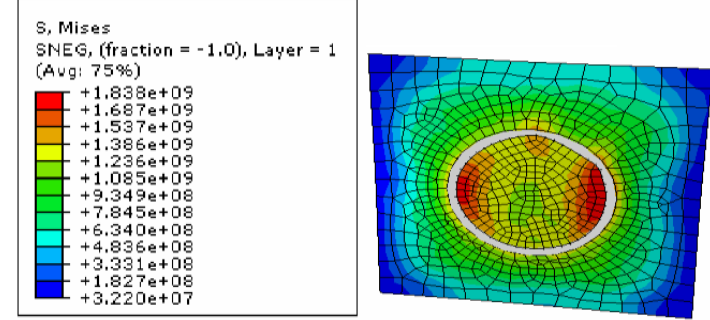

Fig. 5. Von mises stress failure criterion of nanoclay reinforced sample.

\section{CONCLUSIONS}

It was possible to model the nanocomposite impact behavior using the Abaqus software.

The FEM models predicted the failure of the control and the nanocomposite samples due to complete penetration and delamination.

The above results are in line with the experimental results published by the authors earlier.

\section{REFERENCES}

[1] The Internet Journal of Dental Science. (2010, Apr 14). Finite element analysis, A Boon to Dental Research. [Online]. Available: http://www.ispub.com/journal/the_internet_journal_of_dental_scienc e/volume_6_number_2_25/article/finite_element_analysis_a_boon_t o_dental_research.html.

[2] R. Liu and S. S. Quek, The Finite Element Method: A Practical Course, Oxford, Elsevier Science Ltd., 2003.

[3] D. Gay, S. Hoa, and Stephen Tsai, Composite Materials, Design and Applications, France, Paris, CRC Press, 2003.

[4] C. R. Tseng, J. Y. Wu, H. Y. Lee, and F. C. Chang, "Preparation and characterization of polystyrene/clay nanocomposites by free radical polymerization," Journal of Applied Polymer Science, vol. 85, pp. 1370-1377, 2002

[5] M. M. Schwartz, Composite Materials Handbook, USA, New York, McGraw-Hill, 1984.

[6] Z. Hashin and A. Rotem, "A Fatigue criterion for fiber-reinforced materials," Journal of Composite Materials, vol. 7, pp. 448-464, 1973.

[7] Z. Hashin, "Failure criteria for unidirectional fiber composites," Journal of Applied Mechanics, vol. 47, pp. 329-334, 1980

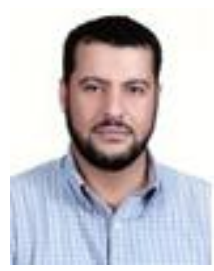

Saud H. Aldajah received his $\mathrm{PhD}$ from the Illinois Institute of Technology in Mechanical Engineering in 2003, He received his MS in mechanical engineering from the the Illinois Institute of Technology in 1998 and BS in mechanical engineering from the Mutah University 1994. He published over forty technical papers and presented many papers at international conferences. He is a co-inventor of a of US patents. Dr. Aldajah's research interests include nanocomposites, modeling of composite materials, tribology and contact mechanics.

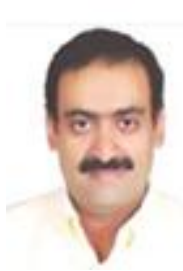

Yousef S. Haik earned his $\mathrm{PhD}$ from the Florida State University in Mechanical Engineering in 1997; He received his MS in mechanical engineering from the University of Iowa in 1994 and received his BS in mechanical engineering from the University of Jordan in 1986. He published and edited nine books and technical proceedings; over two hundred and fifty technical papers and presented many papers at international conferences. $\mathrm{He}$ is the inventor and co-inventor a number of US and International patents. He serves as Editor-in-Chief and on the Editorial Board for a dozen of International Journals. He serves on the review boards for International Research Foundations. He organized and chaired a number of international conferences and symposia. He is a member on a dozen of professional associations. He is a Fellow at the American Society of Mechanical Engineers. 


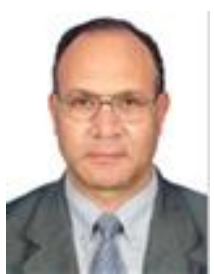

Kamal A. Moustafa received his M.Sc. in mechanical engineering from Ain Shams University, Egypt and he received his $\mathrm{Ph} . \mathrm{D}$. in control engineering from Kyoto University, Japan. He is currently affiliated with the United Arab Emirate University working as a Professor of Mechanical Engineering. Dr. Moustafa research interests include Control Theory, System Modeling and Simulation, Identification and Parameter Estimation of

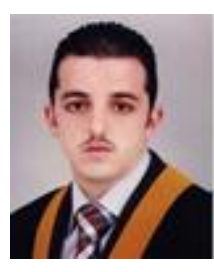

Ammar Alomari received his MS from the United Arab Emirates University in Mechanical Engineering. Currently Mr. Alomari is the Coating and Cutting Tools Manager / Heat Treatment Manager in the Operation Support Department at the Tawazun Precision Industries. His research interests include: Tribological behavior of coated surfaces, FEM, Composite Materials Behavior. 